\title{
Surface plasmon modes for periodic lattices of plasmonic hole waveguides
}

\author{
Ruey-Lin Chern* \\ Institute of Applied Mechanics, National Taiwan University, Taipei 106, Taiwan, Republic of China
}

(Received 21 May 2007; revised manuscript received 9 August 2007; published 15 January 2008)

\begin{abstract}
This study investigates surface plasmon modes for periodic lattices of plasmonic hole waveguides. The interface matching method is proposed to solve the apparently nonlinear eigenvalue problem, with the proper use of the interface condition. It is shown that surface plasmon modes occur for both TE and TM polarizations, and as the off-plane wave number increases, surface plasmon modes for the two polarizations begin to interchange their characteristics. The basic pictures of off-plane surface plasmon modes are explained on a unified basis by examining different types of the interface conditions for TM and TE modes and employing variational arguments based on the Rayleigh quotients for the wave equations. In particular, there exists a TM optical branch which intersects the light line and eventually joins into the surface branches. These features are illustrated by the evolution of surface plasmon mode patterns. For plasmonic hole waveguide structures, the optical branch may exhibit negative group velocities over the whole branch, and the net power flow through the unit cell along the waveguides is shown to direct in the opposite direction of the wave vector.
\end{abstract}

DOI: 10.1103/PhysRevB.77.045409 PACS number(s): 78.67.-n, 78.20.Bh, 42.79.Gn, 42.25.Bs

\section{INTRODUCTION}

Surface plasmons are electromagnetic waves that propagate along the surface of a metal with amplitudes exponentially decaying away from the surface. ${ }^{1}$ These waves come from collective excitations of electric charges in metals, coupled with the electromagnetic fields. ${ }^{2}$ Owing to the field confinement and enhancement, surface plasmons are being explored in optical data storage, ${ }^{3,4}$ biosensing,, 5 light generation, ${ }^{7,8}$ photonic circuit, ${ }^{9,10}$ solar cells, ${ }^{11,12}$ and so forth. By the progress of nanofabrication technologies and microscope measurement techniques, ${ }^{13,14}$ even more applications are proposed.

The properties of surface plasmons strongly depend on the geometry of the metal structures. Particular modes associated with surface plasmons were identified on planar surfaces and gratings, ${ }^{15}$ spherical surfaces, ${ }^{16,17}$ as well as on cylindrical surfaces. ${ }^{18,19}$ Surface plasmons for twodimensional plasmonic crystals have also been investigated in recent years. ${ }^{20-22}$ Once the electromagnetic waves propagate along the off-plane direction, the two-dimensional plasmonic crystals become a periodic lattice of plasmonic waveguides (cf. Fig. 1). On the one hand, surface plasmons on cylindrical surfaces were known to play an important role for light propagation in metallic waveguides. ${ }^{23}$ On the other hand, in recent years, the issue of extraordinary optical transmission through subwavelength hole arrays ${ }^{24}$ has aroused many interests. The transmission characteristics are influenced by the presence of propagating or waveguide modes inside the apertures, ${ }^{25,26}$ as well as by the effect of plasmonic dispersion relation. ${ }^{27}$ This motivates the extension of the previous studies on surface plasmons in plasmonic structures ${ }^{22}$ to those in periodic lattices of plasmonic waveguides.

The most distinguished feature of surface plasmons in periodic structures is a large number of branches of modes intensively gathering around the surface plasma frequency $\omega_{\mathrm{sp}}$. This highly resonant phenomenon comes from strong photon-electron coupling in the metal, and for the in-plane case (where the wave vector lying in the plane formed by the lattice vectors), surface plasmon modes occur only for transverse electric (TE) polarization. This is because for transverse magnetic (TM) polarization, the electric fields are always tangential to the metal surface, and therefore no surface charges exist to support surface plasmons, while for TE polarization, the electric fields possess discontinuous normal components across the interface, giving rise to surface charges for sustaining surface plasma oscillations. For periodic lattices of plasmonic waveguides, surface plasmon characteristics begin to change due to the appearance of the offplane wave number $k_{\|}$(cf. Fig. 1). For this off-plane problem, both TM and TE modes have the electric field components normal to the metal surface (depending on $k_{\|}$), and therefore surface plasmons are feasible in both polarizations.

It has been difficult to solve surface plasmon modes for structures made of dispersive materials due to the nonlinear nature of the underlying eigenvalue problem ${ }^{28}$ and the highly localized feature of surface plasmons at the interface. In previous papers, ${ }^{22,29}$ the interfacial operator approach was developed to circumvent this difficulty and to resolve the inplane surface plasmon modes for plasmonic crystals. This approach has also been applied to investigate surface modes

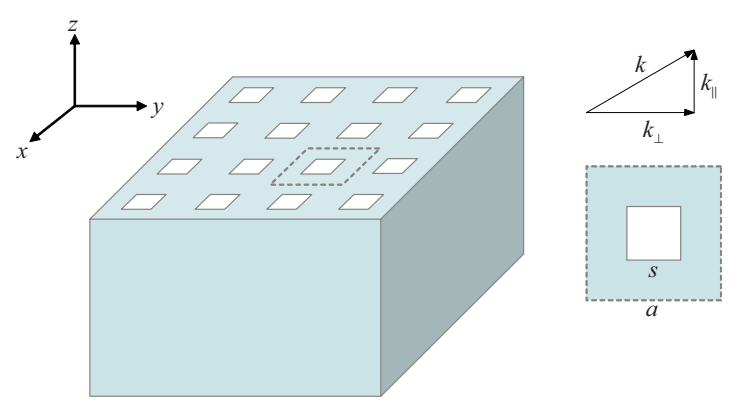

FIG. 1. (Color online) Schematic diagram for a periodic lattice of plasmonic hole waveguides with square cross section. The inplane and off-plane wave numbers, $k_{\perp}$ and $k_{\|}$, respectively, and the unit cell are shown on the right side, where $s$ is the side length of the waveguide and $a$ is the lattice constant. 
for polaritonic crystals ${ }^{30}$ and negative index crystals. ${ }^{31}$ In the present study, the interface matching method is proposed to be applicable to the off-plane surface plasmon modes for periodic lattices of plasmonic waveguides. The basic idea is to separately deal with the wave equations in the strict insides of the dielectric and the plasmonic materials and properly incorporate the interface condition to connect the two different equations. For TE modes, the underlying problem is reformulated as a generalized linear eigensystem, while for TM modes, a quadratic characteristic equation is obtained and further reduced to a generalized linear eigensystem of double size. Both of the eigensystems are then solved by standard eigenvalue solvers.

Surface plasmon modes on plasmonic waveguides constitute the fundamental bases for propagation of the electromagnetic fields along the waveguide structures. It is shown that for periodic lattices of plasmonic waveguides, surface plasmon modes occur for both TE and TM polarizations, owing to the appearance of the off-plane wave number $k_{\|}$. As $k_{\|}$increases, surface plasmon modes for the two polarizations begin to interchange their characteristics. These features are characterized by the off-plane frequency branches of dispersion relations and illustrated by the evolution of surface plasmon mode patterns. For TM polarization, a particular optical branch appears for plasmonic hole waveguides, which is analogous to that in metal film structures. As the unit cell contains enough portion of plasmonic materials, the cutoff frequency of this optical branch will be significantly higher than the surface plasma frequency $\omega_{\mathrm{sp}}$ and the optical branch may exhibit substantial negative group velocities. This reverse nature is further demonstrated by the net power flow through the unit cell along the plasmonic waveguides.

In light of the spirit of the interface matching method, the basic pictures of surface plasmon modes for periodic lattices of plasmonic waveguides are explained on a unified basis by examining different types of the interface conditions and their relations with the surface charges for TM and TE modes. With the help of variational arguments based on the Rayleigh quotients for the wave equations, the highly degenerate nature of off-plane surface plasmon modes is also revealed.

\section{INTERFACE MATCHING METHOD}

The time-harmonic electromagnetic vector wave equations for an inhomogeneous, isotropic, and nonmagnetic medium are written as ${ }^{32}$

$$
\begin{aligned}
& \frac{1}{\varepsilon} \nabla \times \nabla \times \mathbf{E}=\left(\frac{\omega}{c}\right)^{2} \mathbf{E}, \\
& \nabla \times \frac{1}{\varepsilon} \nabla \times \mathbf{H}=\left(\frac{\omega}{c}\right)^{2} \mathbf{H} .
\end{aligned}
$$

For waves propagating along the cylindrical waveguide, for example, in the $z$ direction with $e^{i k_{\|} z}$ dependence (cf. Fig. 1), the wave equations can be expressed (see the Appendix for detail) solely in terms of the off-plane (or parallel) components $E_{\|}$and $H_{\|}$, respectively, for TM and TE modes,

$$
\begin{aligned}
& \nabla_{\perp} \cdot\left(\frac{\varepsilon}{\gamma^{2}} \nabla_{\perp} E_{\|}\right)+\varepsilon E_{\|}=0, \\
& \nabla_{\perp} \cdot\left(\frac{1}{\gamma^{2}} \nabla_{\perp} H_{\|}\right)+H_{\|}=0,
\end{aligned}
$$

where

$$
\begin{gathered}
\gamma^{2}=(\omega / c)^{2} \varepsilon-k_{\|}^{2}, \\
\nabla_{\perp}=\nabla-\hat{\mathbf{z}} \partial / \partial z .
\end{gathered}
$$

Here, TM is referred to the modes without parallel magnetic field $\left(H_{\|}=0\right)$, and TE to the modes without parallel electric field $\left(E_{\|}=0\right)$. The in-plane (or transverse) field components $\mathbf{E}_{\perp}$ and $\mathbf{H}_{\perp}$ are then related to $E_{\|}$through

$$
\begin{gathered}
\mathbf{E}_{\perp}=\frac{i k_{\|}}{\gamma^{2}} \nabla_{\perp} E_{\|}, \\
\mathbf{H}_{\perp}=\frac{i \omega \varepsilon \varepsilon_{0}}{\gamma^{2}} \hat{\mathbf{z}} \times \nabla_{\perp} E_{\|},
\end{gathered}
$$

for TM modes, or related to $H_{\|}$through

$$
\begin{gathered}
\mathbf{E}_{\perp}=-\frac{i \omega \mu_{0}}{\gamma^{2}} \hat{\mathbf{z}} \times \nabla_{\perp} H_{\|}, \\
\mathbf{H}_{\perp}=\frac{i k_{\|}}{\gamma^{2}} \nabla_{\perp} H_{\|},
\end{gathered}
$$

for TE modes. In Eqs. (7)-(10), the time dependence $e^{-i \omega t}$ has been used.

For periodic lattices of waveguide structures, it is sufficient to solve Eqs. (3) and (4) in a unit cell, along with the Bloch conditions applied at the cell boundary,

$$
\begin{gathered}
E_{\|}\left(\mathbf{r}+\mathbf{a}_{i}\right)=e^{i \mathbf{k}_{\perp} \cdot \mathbf{a}_{i}} E_{\|}(\mathbf{r}), \\
H_{\|}\left(\mathbf{r}+\mathbf{a}_{i}\right)=e^{i \mathbf{k}_{\perp} \cdot \mathbf{a}_{i}} H_{\|}(\mathbf{r}),
\end{gathered}
$$

where $\mathbf{k}_{\perp}$ is the in-plane (or Bloch) wave vector (cf. Fig. 1) and $\mathbf{a}_{i}(i=1,2)$ are the lattice translation vectors. In the metal region, the free-electron model

$$
\varepsilon(\omega)=1-\frac{\omega_{p}^{2}}{\omega^{2}}
$$

is used for the dielectric function. Regarding $k_{\|}$and $\mathbf{k}_{\perp}$ as parameters, Eq. (3) for $E_{\|}$together with the boundary condition (11) or Eq. (4) for $H_{\|}$with the condition (12) specifies an eigenvalue problem

$$
\mathcal{L}(\Lambda) \phi=0,
$$

where $\Lambda \equiv \omega^{2} / c^{2}$ is the eigenvalue and $\phi$ is the eigenfunction, which is either $E_{\|}$or $H_{\|}$. Equation (14) does not have a standard format (such as $\mathcal{L} \phi=\Lambda \phi$ ) since the eigenvalue $\Lambda$ appears itself in the differential operator $\mathcal{L}(\Lambda)$. If Eq. (14) is solved by discretization in a straightforward manner, for example, by a finite-difference scheme, one will obtain a nonlinear eigensystem 


$$
\mathbf{A}(\Lambda) \mathbf{x}=0
$$

where $\mathbf{x}$ is the eigenvector consisting of all discrete values of $\phi$ and $\mathbf{A}$ is the matrix system, which is nonlinear in $\Lambda$. Equation (15) belongs to one type of nonlinear eigenvalue problem $^{28}$ that cannot be solved by standard eigenvalue solvers.

However, if the dielectric function $\varepsilon(\omega)$ has some analytical forms, the original nonlinear eigenvalue problem can be reformulated as a linear eigensystem and solved directly by standard eigenvalue solvers. The basic idea is first to deal with the eigensystems [Eqs. (3) and (4)] separately in the strict insides of the dielectric and the metal, so that the dielectric function $\varepsilon$ can be moved out of the differential operator in either region. In the strict inside of the dielectric, Eqs. (3) and (4) become

$$
\begin{aligned}
& \frac{1}{\varepsilon_{d}}\left(-\nabla_{\perp}^{2}+k_{\|}^{2}\right) E_{\|}=\Lambda E_{\|}, \\
& \frac{1}{\varepsilon_{d}}\left(-\nabla_{\perp}^{2}+k_{\|}^{2}\right) H_{\|}=\Lambda H_{\|},
\end{aligned}
$$

where $\varepsilon_{d}$ is the dielectric constant of the dielectric material. In the strict inside of the metal, we have, after rearrangements,

$$
\begin{aligned}
& \left(-\nabla_{\perp}^{2}+k_{\|}^{2}+\Lambda_{p}\right) E_{\|}=\Lambda E_{\|}, \\
& \left(-\nabla_{\perp}^{2}+k_{\|}^{2}+\Lambda_{p}\right) H_{\|}=\Lambda H_{\|},
\end{aligned}
$$

where the free-electron model [Eq. (13)] has been used, with $\Lambda_{p} \equiv \omega_{p}^{2} / c^{2}$. Equations (16) and (18) for $E_{\|}$and Eqs. (17) and (19) for $H_{\|}$in two different regions are then connected, respectively, by the interface conditions

$$
\begin{aligned}
& {\left[\frac{\varepsilon}{\Lambda \varepsilon-k_{\|}^{2}} \frac{\partial E_{\|}}{\partial n}\right]_{S}=0,} \\
& {\left[\frac{1}{\Lambda \varepsilon-k_{\|}^{2}} \frac{\partial H_{\|}}{\partial n}\right]_{S}=0,}
\end{aligned}
$$

where $[\cdots]_{S}$ is the discontinuity across the interface $S$ between the dielectric and the metal and $\partial / \partial n$ denotes the derivative in the direction normal to the interface. The interface conditions (20) and (21) are obtained by integrating both sides of the eigensystem [Eqs. (3) and (4)] over a thin box located on the interface and taking the limit as the box height goes to zero. Denoting + and - the dielectric and the metal sides, respectively, of the interface, Eqs. (20) and (21) are written as

$$
\begin{aligned}
& \left.\frac{\Lambda \varepsilon_{d}}{\Lambda \varepsilon_{d}-k_{\|}^{2}} \frac{\partial E_{\|}}{\partial n}\right|_{+}=\left.\frac{\Lambda-\Lambda_{p}}{\Lambda-\Lambda_{p}-k_{\|}^{2}} \frac{\partial E_{\|}}{\partial n}\right|_{-}, \\
& \left.\frac{1}{\Lambda \varepsilon_{d}-k_{\|}^{2}} \frac{\partial H_{\|}}{\partial n}\right|_{+}=\left.\frac{1}{\Lambda-\Lambda_{p}-k_{\|}^{2}} \frac{\partial H_{\|}}{\partial n}\right|_{-}
\end{aligned}
$$

The next step is to reformulate Eqs. (22) and (23) as characteristic equations of the eigensystem. Figure 2 shows a sche-



FIG. 2. (Color online) Schematic diagram of the unit cell for a periodic lattice of plasmonic waveguides. The shaded area corresponds to the metal region. Eight types of interface points are denoted by (1)-(8).

matic diagram of the unit cell for a periodic lattice of plasmonic waveguides. According to the geometric position of the interface between the dielectric and the metal, there are eight types of interface points. Four of them appear at sides [right, left, top, and bottom, denoted by (1), (2), (3), and (4), respectively], and the other four appear at corners [top right, top left, bottom right, and bottom left, denoted by (5), (6), (7), and (8), respectively].

\section{A. Transverse magnetic modes}

Let $(i, j)$ denote the interface point, for example, of the type (1). Using one-sided difference to discretize the interface condition (22) for $E_{\|}$in either region of the dielectric and the metal, one obtains a quadratic equation

$$
\Lambda^{2} I_{i, j}^{(2)}-\Lambda I_{i, j}^{(1)}-I_{i, j}^{(0)}=0
$$

where

$$
\begin{gathered}
I_{i, j}^{(2)}=\varepsilon_{d}\left(-E_{i-1, j}+2 E_{i, j}-E_{i+1, j}\right) \\
I_{i, j}^{(1)}=-\left(\varepsilon_{d} \Lambda_{p}+\varepsilon_{d} k_{\|}^{2}\right) E_{i-1, j}+\left[2 \varepsilon_{d} \Lambda_{p}+\left(\varepsilon_{d}+1\right) k_{\|}^{2}\right] E_{i, j} \\
-\left(\varepsilon_{d} \Lambda_{p}+k_{\|}^{2}\right) E_{i+1, j}, \\
I_{i, j}^{(0)}=k_{\|}^{2} \Lambda_{p}\left(-E_{i, j}+E_{i+1, j}\right) .
\end{gathered}
$$

For clarity, the subscript in $E_{\|}$has been omitted. Expressions of the interface conditions for types (2)-(8) can also be obtained in a similar way.

In the strict insides of the dielectric and the metal, central finite difference is used to discretize Eqs. (16) and (18). At an interior point $(i, j)$, they are written as

$$
\begin{gathered}
\Lambda^{2} E_{i, j}-\Lambda\left[\frac{1}{\varepsilon_{d}}\left(L_{i, j}+k_{\|}^{2} E_{i, j}\right)\right]=0, \\
\Lambda^{2} E_{i, j}-\Lambda\left[L_{i, j}+\left(\Lambda_{p}+k_{\|}^{2}\right) E_{i, j}\right]=0,
\end{gathered}
$$

where 


$$
L_{i, j}=\frac{1}{h^{2}}\left(-E_{i-1, j}-E_{i, j-1}+4 E_{i, j}-E_{i+1, j}-E_{i, j+1}\right),
$$

with $h$ being the spacing of discretization. Equation (24) for the interface and Eqs. (25) and (26) for the strict insides are then combined together to give a matrix system

$$
\left(\Lambda^{2} \mathbf{B}-\Lambda \mathbf{A}-\mathbf{C}\right) \mathbf{x}=0,
$$

where $\mathbf{x}$ is the eigenvector consisting of all values of $E_{i, j}$ and $\mathbf{B}, \mathbf{A}$, and $\mathbf{C}$ are square matrices. Unlike Eq. (15), the entries of $\mathbf{B}, \mathbf{A}$, and $\mathbf{C}$ are now independent of the eigenvalue $\Lambda$. Introducing an auxiliary vector $\mathbf{y}=\boldsymbol{\Lambda} \mathbf{x}$, Eq. (28) is recast into the following form:

$$
\left[\begin{array}{ll}
\mathbf{0} & \mathbf{I} \\
\mathbf{C} & \mathbf{A}
\end{array}\right]\left[\begin{array}{l}
\mathbf{x} \\
\mathbf{y}
\end{array}\right]=\Lambda\left[\begin{array}{ll}
\mathbf{I} & \mathbf{0} \\
\mathbf{0} & \mathbf{B}
\end{array}\right]\left[\begin{array}{l}
\mathbf{x} \\
\mathbf{y}
\end{array}\right] .
$$

This is a generalized eigensystem $\mathbf{A}^{\prime} \mathbf{x}^{\prime}=\mathbf{\Lambda} \mathbf{B}^{\prime} \mathbf{x}^{\prime}$, which can be solved by standard eigenvalue solvers at the expense of doubling the matrix size.

\section{B. Transverse electric modes}

Likewise, discretization of Eq. (23) for $H_{\|}$at an interface point $(i, j)$ of the type (1) gives a linear equation

$$
\begin{gathered}
-\left(\Lambda_{p}+k_{\|}^{2}\right) H_{i-1, j}+\left(\Lambda_{p}+2 k_{\|}^{2}\right) H_{i, j}-k_{\|}^{2} H_{i+1, j} \\
=\Lambda\left[-H_{i-1, j}+\left(1+\varepsilon_{d}\right) H_{i, j}-\varepsilon_{d} H_{i+1, j}\right] .
\end{gathered}
$$

The subscript in $H_{\|}$has also been omitted for clarity. Expressions of the interface conditions for types (2)-(8) can be obtained in a similar way. In the strict insides of the dielectric and the metal, central finite differences of Eqs. (17) and (19) for an interior point $(i, j)$ give, respectively,

$$
\begin{gathered}
\frac{1}{\varepsilon_{d}}\left(M_{i, j}+k_{\|}^{2} H_{i, j}\right)=\Lambda H_{i, j}, \\
M_{i, j}+\left(\Lambda_{p}+k_{\|}^{2}\right) H_{i, j}=\Lambda H_{i, j},
\end{gathered}
$$

where

$$
M_{i, j}=\frac{1}{h^{2}}\left(-H_{i-1, j}-H_{i, j-1}+4 H_{i, j}-H_{i+1, j}-H_{i, j+1}\right) .
$$

Combining Eqs. (29)-(31), one obtains a generalized eigensystem

$$
\mathbf{A x}=\Lambda \mathbf{B x}
$$

where $\mathbf{x}$ is the eigenvector consisting of all values of $H_{i, j}$ and $\mathbf{A}$ and $\mathbf{B}$ are square matrices independent of the eigenvalue $\Lambda$. This equation can be solved by standard eigenvalue solvers.

\section{Interface condition}

The crucial step of the present approach is the proper incorporation of the interface conditions into the wave equations. In fact, the interface condition comes from degradation of the wave equation at the boundary between two different media. In the continuous sense, the function of interface con- dition is already implied in the wave equation, and it is not necessary to include this condition explicitly. However, discretization of the wave equation across the interface may give rise to inaccurate and unstable results. This is due to the nondifferentiability of the solution of surface plasmon modes at the interface and is more severe for plasmonic structures where the dielectric function becomes negative. In fact, this is the typical feature of surface plasmon modes that needs to be resolved.

Explicit use of the interface condition comes to circumvent this difficulty. This not only serves as a means to solve surface plasmon modes but also provides a different perspective to examine properties of these modes. It is known that for in-plane problems $\left(k_{\|}=0\right)$, only TE modes sustain surface plasmons. However, the wave equations for TE and TM modes in the strict inside of either the metal or the dielectric region are the same [cf. Eqs. (16) and (17) or (18) and (19)], while the only difference is the interface condition [cf. Eqs. (20) and (21)] between the two regions. In this regard, the type of interface condition is essential to account for the existence of surface plasmons, and the proper use of this condition may reveal the physical picture of surface plasmon modes.

For off-plane problems, both TE and TM modes sustain surface plasmons (as will be shown in the next section), depending on the off-plane wave number $k_{\|}$. This is also manifested on the interface conditions (20) and (21) for $k_{\|} \neq 0$. Besides, surface plasmon modes for TE and TM polarizations gradually interchange their characteristics as $k_{\|}$increases. At the two extremes, $k_{\|}=0$ and $k_{\|}=\infty$, the interface conditions have very opposite natures as $k_{\|}^{2}$ appears in their denominators. This point will be discussed in detail in the next section.

\section{RESULTS AND DISCUSSION}

The present study investigates surface plasmon modes for periodic lattices of plasmonic hole waveguides. For periodic structures made of plasmonic materials, there are basically two coupling or dispersion mechanisms responsible for the properties of surface plasmon modes. The first one is the material dispersion due to the electron motion in the metal. This is considered by using the free-electron model [Eq. (13)] for the dielectric function $\varepsilon(\omega)$. The second one is the structure dispersion due to the periodic scattering by the lattice. This is taken into account by applying the Bloch conditions (11) and (12). The interplay of the two distinct dispersions gives rise to special properties of surface plasmon modes pertaining to periodic structures, in particular, for plasmonic waveguides. This is resolved by employing the interface matching method proposed in the previous section. In all subsequent results, the bulk plasma frequency $\omega_{p}$ in the free-electron model [Eq. (13)] is given as $\omega_{p} a / 2 \pi c=1$. Unless otherwise specified, the dielectric material filled inside the hole has the dielectric constant $\varepsilon_{d}=1$.

\section{A. Surface plasmon modes}

For periodic structures, surface plasmon modes are eigenmodes associated with surface plasmons. These modes con- 


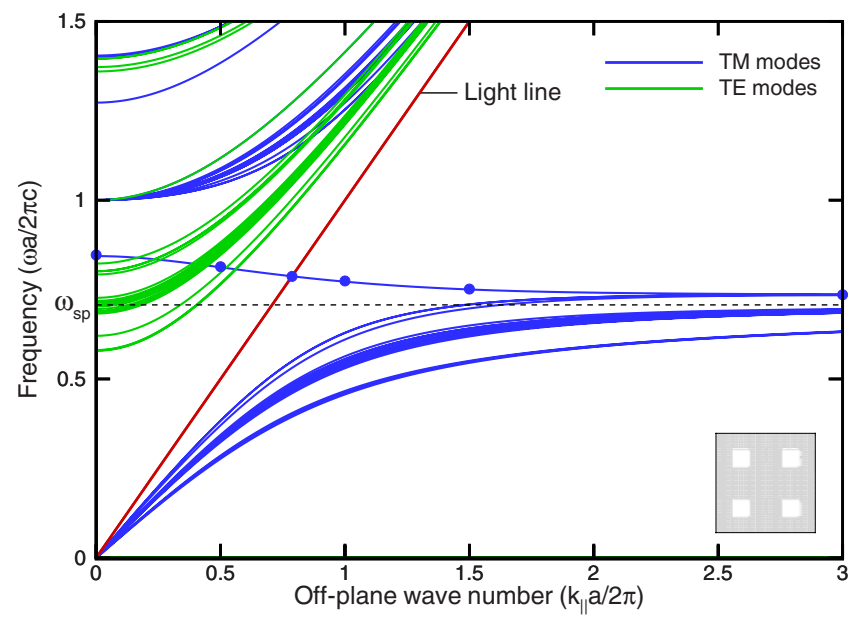

FIG. 3. (Color online) Off-plane dispersion relations for a periodic lattice [at the point $\left.\Gamma, \mathbf{k}_{\perp}=(0,0)\right]$ of plasmonic hole waveguides with square cross section of side length $s / a=0.4$. The inset shows four $(2 \times 2)$ unit cells of the structures.

stitute the fundamental bases for propagation of the electromagnetic fields along plasmonic waveguides. The important features of surface plasmon modes are characterized by the off-plane dispersion relations, in which the eigenfrequencies vary with the in-plane and off-plane wave vectors, where variations of the electromagnetic fields in time and in space are correlated.

\section{Dispersion relation}

Figure 3 shows the off-plane dispersion relations for a periodic lattice [at the point $\left.\Gamma, \mathbf{k}_{\perp}=(0,0)\right]$ of plasmonic hole waveguides with square cross section of side length $s / a$ $=0.4$. It is shown that a large number of branches of surface plasmon modes (in green color) appear for TE polarization at $k_{\|}=0$. They intensively gather around the surface plasma frequency $\omega_{\text {sp }}$ given by

$$
\omega_{\mathrm{sp}}=\frac{\omega_{p}}{\sqrt{1+\varepsilon_{d}}},
$$

which is the same frequency as for planar surfaces. ${ }^{15}$ No branches of surface plasmon modes are observed for TM polarization at $k_{\|}=0$. This is consistent with the results of in-plane surface plasmon modes for plasmonic crystals. ${ }^{22}$ For TE polarization, the electric fields lie completely in the transverse plane, and normal electric field components (to the metal surface) give rise to strong surface charges, which are the source of surface plasmons. However, as the off-plane wave number $k_{\|}$increases from zero, the electric fields turn aside from the normal to tangential direction. The frequencies of surface plasmon modes begin to deviate from $\omega_{\text {sp }}$ to higher values. As $k_{\|}$continue to increase, normal electric field components become even smaller, and the TE branches gradually lose their surface nature. For larger $k_{\|}$, these branches of modes become parallel to the light line $(\omega$ $=k_{\|} c$ ). In the large $k_{\|}$limit, normal components of the electric fields vanish and these modes grow into bulk modes.
Meanwhile, it is shown in Fig. 3 that a large number of branches of TM modes (in blue color) emerge from $\omega=0$ at $k_{\|}=0$ (same as the in-plane static mode). Their frequencies increase with $k_{\|}$and mostly remain below the surface plasma frequency $\omega_{\text {sp. }}$. In the large $k_{\|}$limit, they develop into surface modes with frequencies approaching $\omega_{\mathrm{sp}}$. By the presence of off-plane propagation, the electric fields for TM modes are no longer kept parallel to the metal surface. Normal components of the electric fields give rise to surface charges, and therefore surface plasmons begin to appear. Basically, these surface branches share the common features of those in semi-infinite metal, ${ }^{15}$ metal films, ${ }^{33}$ one-dimensional plasmonic crystals, ${ }^{22}$ and metallic coaxial waveguides. ${ }^{34}$ For semi-infinite metal, there is only one surface branch, while for metal films, one-dimensional plasmonic crystals, and metallic coaxial waveguides, two surface branches were observed. In the present problem, there are a large number of surface branches that constitute a surface frequency bunch. The number of branches in this bunch is expected to be infinite for the periodic structure with infinite extent may lead to infinite degeneracy of the corresponding surface modes. In the continuous sense, these branches form a frequency band in the frequency-wave number space.

It is noted that for sufficiently large $k_{\|}$, the off-plane surface plasmon modes vary very little with the in-plane wave vector $\mathbf{k}_{\perp}$. They are nearly degenerate since oscillations in the nearby waveguides become decoupled. Besides, retardation effects introduced through coupling of the transverse fields are now unimportant. This is because these surface branches are far from the light line at large $k_{\|}$, that is, $\omega / k_{\|}$ $\ll c$ in both the dielectric and the metal. The ratio of the distance over which electric charges are transferred to the time required for such a transport with velocities of the electromagnetic waves is much smaller than the speed of light in either region. ${ }^{33}$ Accordingly, the electrostatic theory ${ }^{35}$ is adequate for describing this nonretarded phenomenon. The other branches of TM modes are located at the other side (the upper left) of the light line. They are bulk in nature and appear with anticrossing interaction to those TM surface branches (with respect to the light line).

\section{Optical branch}

In Fig. 3, there exists a crossing scheme between the TM optical branch and the light line. This branch begins with a cutoff frequency at $k_{\|}=0$, intersects the light line at an intermediate value of $k_{\|}$, and approaches the TM surface branches. Eventually, this branch develops into a typical surface plasmon mode and joins the bunch of surface branches from above in the large $k_{\|}$limit. The nonretarded property of surface plasmon branches at large $k_{\|}$(addressed in the previous paragraph) also applies to this particular optical branch.

For $\varepsilon_{d}=1$, the light line $\left(\omega=k_{\|} c\right)$ is also a solution to the dispersion relations for both TM and TE modes [at the point $\left.\Gamma, \mathbf{k}_{\perp}=(0,0)\right]$. The mode structures have the same typical feature of static modes $(\omega=0)$ for the in-plane case $\left(k_{\|}=0\right)$, where the field is constant over the dielectric region and decaying in the metal..$^{22}$ For the off-plane case $\left(k_{\|} \neq 0\right)$, the same type of solution appears as $\omega=k_{\|} c$ is satisfied. In this situation, Eqs. (16)-(19) lead to solutions with the same form 


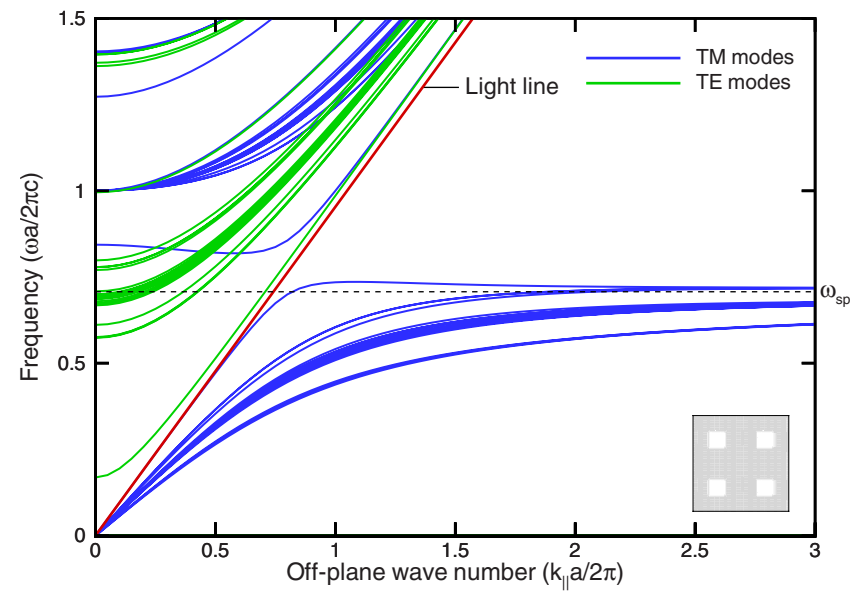

FIG. 4. (Color online) Off-plane dispersion relations for a periodic lattice [at the point $\left.\Gamma, \mathbf{k}_{\perp}=(0,0)\right]$ of plasmonic hole waveguides with square cross section of side length $s / a=0.4$. The dielectric material filled in the hole has $\varepsilon_{d}=1.1$.

of static modes in the in-plane case [with the corresponding mode structure shown in Fig. 5(c) in the next paragraph]. However, for $\varepsilon_{d} \neq 1$, such solutions do not exist. Figure 4 shows the dispersion relations for the same waveguide structure in Fig. 3, with the dielectric material filled in the hole having $\varepsilon_{d}=1.1$. In this case, the TM optical branch no longer intersects the light line but interacts with an anticrossing manner.

To illustrate the evolution of surface plasmon modes on the TM optical branch (for $\varepsilon_{d}=1$ ), Fig. 5 shows the mode patterns ( $E_{\|}$in magnitude) at the marked positions in Fig. 3, where $k_{\|} a / 2 \pi=0,0.5,0.8,1,1.5$, and 3 . All the field amplitudes have been normalized to have the maximum of unity, that is, $\left|E_{\|}\right|_{\max }=1$. Figure 5(a) corresponds to the in-plane cutoff frequency $\omega a / 2 \pi c=0.844$, where the field spreads over the whole region including the hole and the metal. As $k_{\|}$ increases, the field gradually concentrates on the hole [cf. Fig. 5(b)]. At the intersecting point (with the light line), the field is uniform in the hole area and decays in the metal, as shown in Fig. 5(c). For larger $k_{\|}$, the field decays in both the hole and metal regions [cf. Fig. 5(d)] and becomes evanescent [cf. Fig. 5(e)]. At sufficiently large $k_{\|}$, the field almost vanishes except near the interface and the field pattern of $E_{\|}$ shows a very sharp profile at the interface [cf. Fig. 5(f)]. This is the most distinguished feature of surface plasmon modes, and in the present approach it only takes a few grid points to resolve this feature. Note that the present results share similar features of surface plasmon modes in plasmonic crystals, ${ }^{22}$ surface phonon modes in polaritonic crystals, ${ }^{30}$ as well as magnetic surface plasmon modes in negative index crystals. ${ }^{31}$

\section{Negative group velocity}

It is noted that the optical branch in Fig. 3 exhibits negative slopes over the whole branch, which leads to negative group velocities in the off-plane direction, that is, $\left(v_{g}\right)_{\|}$ $=\partial \omega / \partial k_{\|}<0$. Negative group velocity has been found in metal film structures ${ }^{33}$ and serves as a photonic approach to making a material with a negative index of refraction. ${ }^{36}$ More recently, all-angle negative refraction based on surface plasmon waves has also been demonstrated. ${ }^{37}$ Negative group velocity also indicates that power flow of the electromagnetic field is opposite to the phase velocity along the waveguides. ${ }^{38}$ In the present problem, this fact can be understood from the parallel component of the time-averaged Poynting vector (for TM modes),

$$
\left\langle S_{\|}\right\rangle=\frac{1}{2} \operatorname{Re}\left[\mathbf{E}_{\perp} \times \mathbf{H}_{\perp}^{*} \cdot z\right]=\frac{k_{\|} \omega \varepsilon \varepsilon_{0}}{2 \gamma^{4}}\left|\nabla_{\perp} E_{\|}\right|^{2},
$$

where Eqs. (7) and (8) for $\mathbf{E}_{\perp}$ and $\mathbf{H}_{\perp}$, respectively, have been used. Since $\gamma^{4} \geqslant 0,\left\langle S_{\|}\right\rangle$always has the same sign as $k_{\|}$ in the dielectric region, while in the metal region, the direction of $\left\langle S_{\|}\right\rangle$depends on the frequency. For $\omega<\omega_{p}, \varepsilon<0$ and $\left\langle S_{\|}\right\rangle$is antiparallel to $k_{\|}$. It is then possible that the net timeaveraged power flow through (and normal to) the unit cell along the waveguides, $\left\langle P_{\|}\right\rangle=\int_{\text {cell }}\left\langle S_{\|}\right\rangle d a$, may direct in the opposite direction of $k_{\|}$. This can be attained if the unit cell of the waveguide arrays contains sufficiently large portion of the metal, and therefore more power flows in the metal region than in the dielectric. For this reason, the hole waveguide structures are more likely to achieve this phenomenon.

To illustrate the reverse direction of the net power flow, Fig. 6 shows the vector plots of the electric field, magnetic field, and Poynting vectors at $k_{\|} a / 2 \pi=0.5$ for a periodic lattice [at the point $\left.M, \mathbf{k}_{\perp} a / 2 \pi=(0.5,05)\right]$ of plasmonic hole waveguides with square cross section of side length $s / a$ $=0.4$ for TM modes, where $\omega a / 2 \pi c=0.845$. The upper and lower graphs correspond to real and imaginary parts, respectively. It is shown that the real parts of electric fields [Fig. 6(a)] point in the same direction along the waveguide in the whole region, while the imaginary parts orient radially in the transverse plane but with opposite directions in the metal and the dielectric. For the magnetic fields [Fig. 6(b)], only transverse components remain $\left(H_{\|}=0\right.$ for TM modes). They are purely imaginary and circulating around the cell center. It follows that the parallel component of the time-averaged Poynting vectors $\left\langle S_{\|}\right\rangle$[upper graph of Fig. 6(c)] direct upward in the dielectric and downward in the metal.

The net power flow through the unit cell along the plasmonic waveguides can further be characterized by the energy velocity, $\left(v_{e}\right)_{\|}=\left\langle P_{\|}\right\rangle /\langle U\rangle$, where $\langle U\rangle=\int_{\text {cell }}\langle u\rangle d a$ is the total time-averaged field energy over the unit cell per unit length of the waveguide and $u$ is the field energy density, which for dispersive materials ${ }^{39}$

$$
u=\frac{1}{2} \varepsilon_{0} \operatorname{Re}\left[\frac{d(\omega \varepsilon)}{d \omega}\right]|\mathbf{E}|^{2}+\frac{1}{2} \mu_{0} \operatorname{Re}\left[\frac{d(\omega \mu)}{d \omega}\right]|\mathbf{H}|^{2} .
$$

Based on the free-electron model [Eq. (13)], the timeaveraged field energy density is then given by

$$
\langle u\rangle=\frac{1}{4} \varepsilon_{0}\left(1+\frac{\omega_{p}^{2}}{\omega^{2}}\right)|\mathbf{E}|^{2}+\frac{1}{4} \mu_{0}|\mathbf{H}|^{2} .
$$

In Fig. 6(c), the distribution of $\langle u\rangle$ is shown on the lower graph. Integrating $\left\langle S_{\|}\right\rangle$and $\langle u\rangle$ over the unit cell, the energy velocity is calculated to give $\left(v_{e}\right)_{\|}=(-12.1 / 66.6) c=-0.182 c$. 

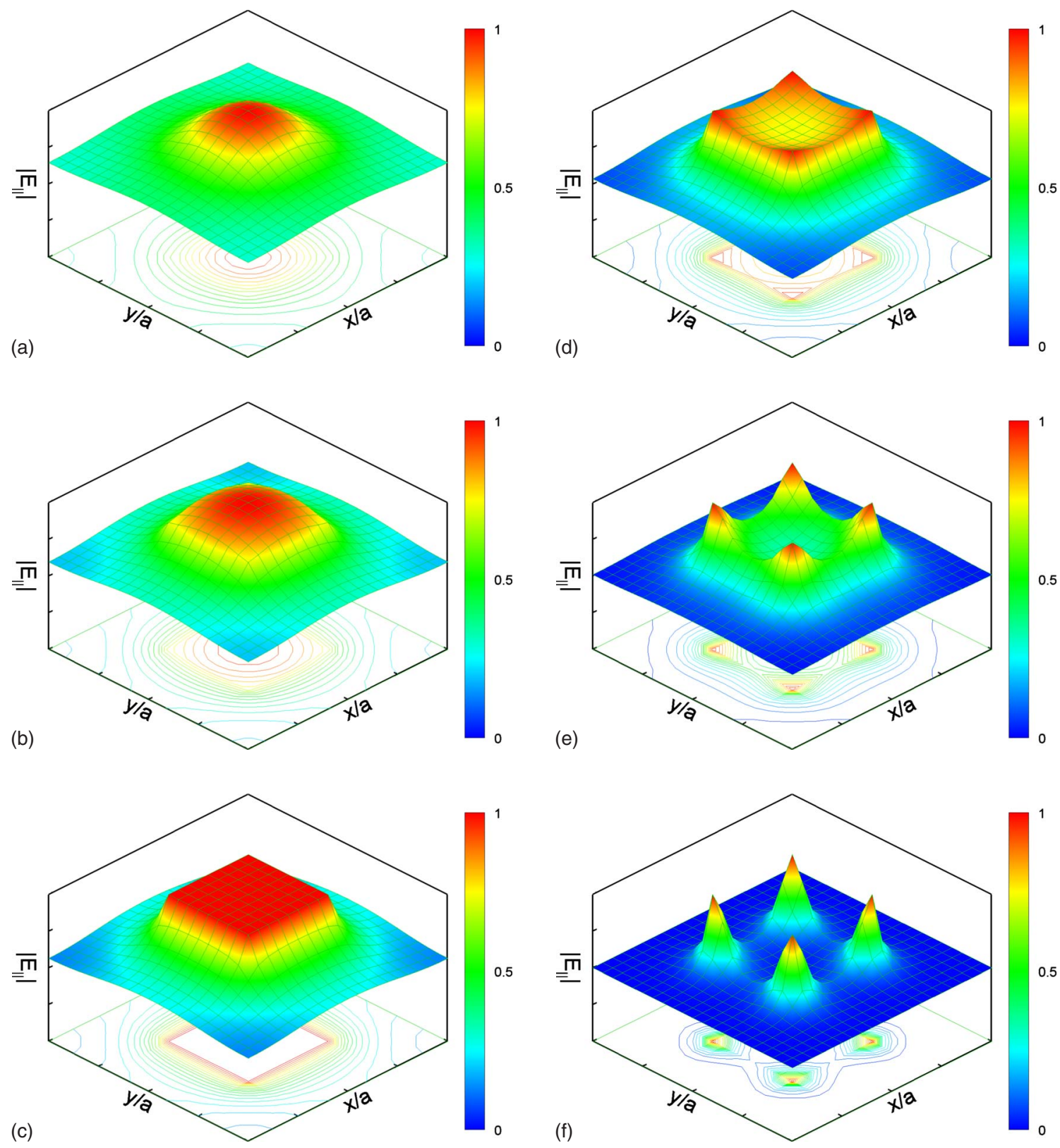

FIG. 5. (Color online) TM mode structures $\left(E_{\|}\right.$in magnitude) for a periodic lattice [at the point $\Gamma$, $\left.\mathbf{k}_{\perp}=(0,0)\right]$ of plasmonic hole waveguides with square cross section of side length $s / a=0.4$, where $k_{\|} a / 2 \pi$ are (a) 0 , (b) 0.5 , (c) 0.8 , (d) 1 , (e) 1.5 , and (f) 3 .

This value is consistent with the slope of dispersion relation at the corresponding point $\left(k_{\|} a / 2 \pi=0.5\right):\left(v_{g}\right)_{\|}=\partial \omega / \partial k_{\|}=$ $-0.186 c$. As is well known, the group velocity in periodic structures is equal to the average energy velocity over the unit cell. ${ }^{40,41}$

It is noted that the group velocity being antiparallel to the phase velocity may correspond to two different cases. Assume that the plasmonic structure has an effective refractive index $n$, which is a function of frequency $\omega$. The phase velocity $v_{p}$ is

$$
v_{p}=\frac{\omega}{k}=\frac{c}{n}
$$

and the group velocity $v_{g}$ is given as

$$
v_{g}=\frac{\partial \omega}{\partial k}=\frac{c}{n+\omega \frac{d n}{d \omega}}
$$

The group velocity is then related to the phase velocity by

$$
v_{g}=\frac{v_{p}}{\alpha}, \quad \alpha=1+\frac{\omega}{n} \frac{d n}{d \omega} .
$$

If $\alpha<0, v_{g}$ and $v_{p}$ have opposite signs. This can be attained when either (1) $v_{g}<0$ and $v_{p}>0$ or (2) $v_{g}>0$ and $v_{p}<0$. In case (1), the group velocity has a negative value, indicating that the pulse propagates in the backward direction within the medium. This phenomenon has been concluded 

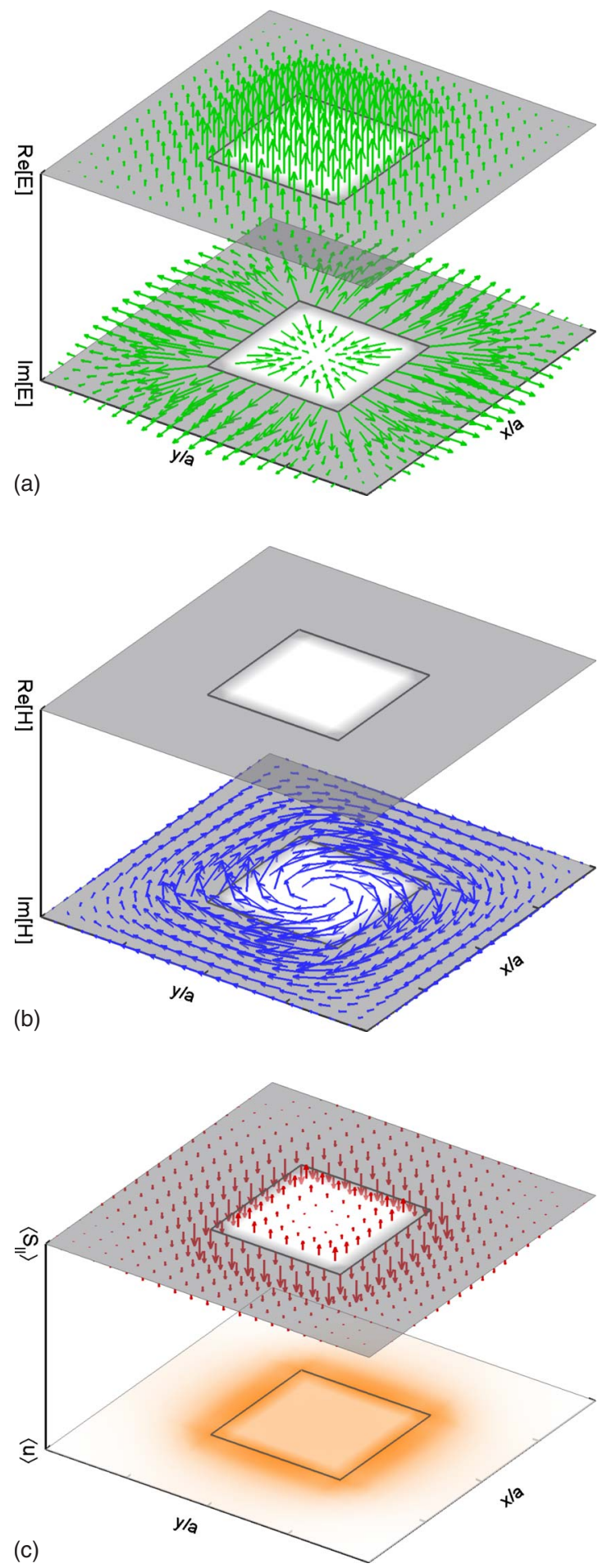

FIG. 6. (Color online) Vector plots of (a) real and imaginary parts of $\mathbf{E}$, (b) real and imaginary parts of $\mathbf{H}$, and (c) $\left\langle S_{\|}\right\rangle$and $\langle u\rangle$ at $k_{\|} a / 2 \pi=0.5$ for a periodic lattice [at the point $M, \mathbf{k}_{\perp} a / 2 \pi$ $=(0.5,05)]$ of plasmonic hole waveguides with square cross section of side length $s / a=0.4$ for TM modes, where $\omega a / 2 \pi c=0.845$ and the energy velocity $\left(v_{e}\right)_{\|}=-0.182 c$.

physical $^{42}$ and the backward pulse propagation was observed in the recent experiment. ${ }^{43}$ Referring to Eq. (38), $v_{g}<0$ occurs in the medium with anomalous dispersion such that $n$ $+\omega \frac{d n}{d \omega}<0$. If the effective refractive index decreases rapidly enough with frequency in a certain range, the group velocity $v_{g}$ can be negative.

On the other hand, in case (2), the phase velocity has a negative value, which means that the pulse still propagates away from the source, while the phase fronts run backward. This will happen in media where the effective refractive index $n<0$ in some frequency range and has been observed in left-handed metamaterials. ${ }^{44}$ It is therefore feasible that the negative slope of dispersion curve corresponds to a negative phase velocity but with a positive group velocity. ${ }^{45} \mathrm{~A}$ general condition for the phase velocity being oppositely directed to the power flow has been derived, ${ }^{46}$ in which the dissipative loss was taken into account. Since the power flow is always in the direction of exponential decrease of the field amplitudes in a passive medium as required by causality, ${ }^{46}$ proper implementation of losses ${ }^{47}$ would help clarify distinction between the two different cases originated from the negative slope of dispersion curve.

\section{Cutoff behavior}

It is also noted that in Fig. 3, the optical branch has a significant portion above (and to the left of) the light line with phase velocity larger than the velocity of light. In metal film structures, the corresponding modes acquire a radiative nature ${ }^{33}$ and the externally incident light from air can therefore couple to this band if the metal region has finite thickness. ${ }^{48}$ For periodic lattices of plasmonic waveguides, these properties are still applicable. In addition, this optical branch has a cutoff (at $k_{\|}=0$ ) which is similar to the role of the cutoff frequency $\omega_{c}$ for the waveguide. It is known that $\omega_{c}$ for a perfectly conducting hole waveguide with radius $r$ is given as ${ }^{49}$

$$
\omega_{c}=\frac{p_{11}^{\prime} c}{r \sqrt{\varepsilon_{d}}},
$$

where $p_{11}^{\prime} \approx 1.841$ is the first root of $J_{m}^{\prime}\left(p_{m n}^{\prime}\right)=0$ for $m=1$ and $\varepsilon_{d}$ is the dielectric constant of the dielectric material inside the hole. The cutoff frequency is inversely proportional to the hole size. This reflects the fact that electromagnetic fields are completely expelled from perfectly conducting materials. For a plasmonic waveguide, the relation between the cutoff frequency and the hole size was substantially altered. The cutoff frequency $\omega_{c}$ is implicitly determined by the following equation: ${ }^{18,27}$

$$
\frac{1}{k_{d}} \frac{J_{1}^{\prime}\left(k_{d} r\right)}{J_{1}\left(k_{d} r\right)}-\frac{1}{k_{m}} \frac{H_{1}^{(1)^{\prime}}\left(k_{m} r\right)}{H_{1}^{(1)}\left(k_{m} r\right)}=0,
$$

where $k_{d}=\left(\omega_{c} / c\right) \sqrt{\varepsilon_{d}}, k_{m}=\left(\omega_{c} / c\right) \sqrt{\varepsilon_{m}}$, and $\varepsilon_{m}$ is the dielectric constant of the metal. It has been shown that for smaller holes, $\omega_{c}$ is greatly reduced, while for larger holes, $\omega_{c}$ is slightly affected. ${ }^{27}$ In short, $\omega_{c}$ is less sensitive to the change of the size for a plasmonic waveguide than a perfectly conducting one. This is due to the partial (though very little) penetration of the electromagnetic fields into the metal.

For periodic lattices of plasmonic waveguides, coupling between nearby waveguides may further modify the cutoff behavior. Nevertheless, the qualitative trend of the cutoff fre- 


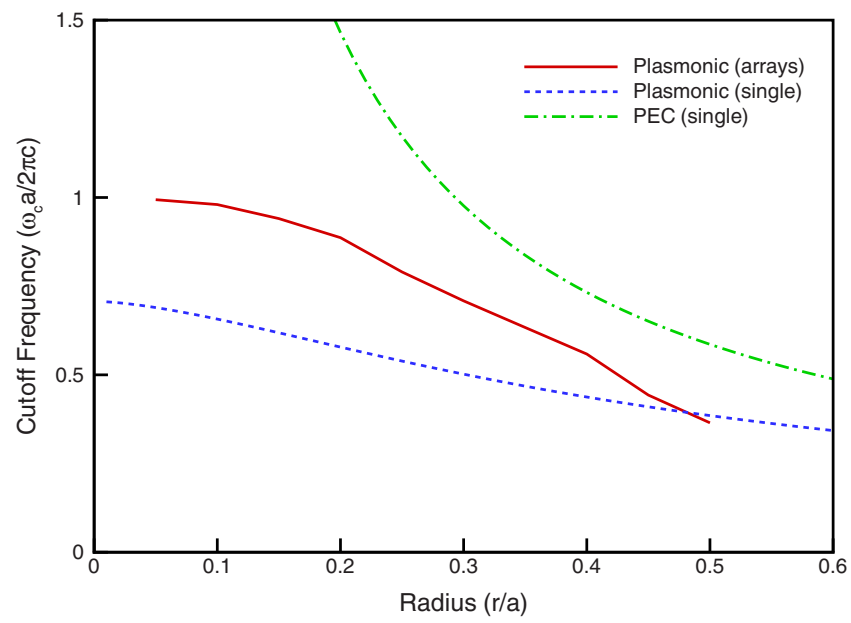

FIG. 7. (Color online) Cutoff frequencies of the TM optical branch for a periodic lattice [at the point $\left.\Gamma, \mathbf{k}_{\perp}=(0,0)\right]$ of plasmonic hole waveguides with circular cross section.

quency of the optical branch for plasmonic waveguide arrays is similar to that for single plasmonic waveguides. Figure 7 shows the cutoff frequency of the TM optical branch for a periodic lattice of plasmonic hole waveguides with circular cross section. The cutoff frequencies for a single perfectly conducting waveguide and a single plasmonic waveguide are also shown for comparison. In the moderate to large hole region (up to $r / a=0.5$ where the holes contact with each other), the cutoff frequency decreases more rapidly with increasing the radius $r$, while in the small hole region, $\omega_{c}$ comes close to the bulk plasma frequency $\omega_{p}$ and becomes saturated as the radius is small enough. This trend is in agreement with the similar behavior on metal film structures, where an analytic expression for the cutoff frequency can be obtained. ${ }^{33}$ For sufficiently small holes, most parts of the unit cell consist of plasmonic materials, and the cutoff frequency of the optical branch approaches $\omega_{p}$.

On the other hand, if the metal portion is reduced, the cutoff frequency of the optical branch is decreased and may even lie below the surface plasma frequency $\omega_{\mathrm{sp}}$. For a moderate size of hole, $\omega_{c}$ can therefore be very close to $\omega_{\mathrm{sp}}$. Figure 8 shows that for the hole size $s / a=0.54$, the whole optical branch is nearly flat with very slow group velocities. ${ }^{50,51}$ This is an important attribute that can be used to design very high- $Q$ cavities of nanoscale, ${ }^{52}$ and enhance the nonlinear effect for reducing the required operational power in photonic devices. ${ }^{53}$ In addition, the dispersionless (that is, independent of $k_{\|}$) band indicates omnidirectional resonance, where the resonance occurs approximately at the same frequency for all incident angles. ${ }^{54}$

\section{Effect of loss}

In real systems, the loss due to intrinsic damping of metals has to be considered, in particular, in the optical range. The effect of loss may cause the dispersion curve to bend back toward the light line instead of increasing asymptotically to the surface plasmon energy at infinite momentum. ${ }^{55}$ This phenomenon has been explained in the measurement

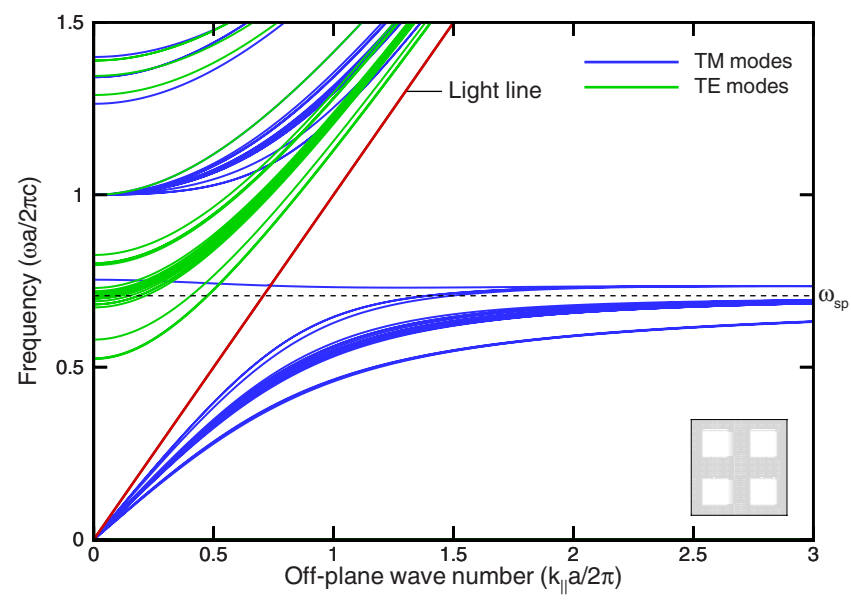

FIG. 8. (Color online) Off-plane dispersion relations for a periodic lattice [at the point $\left.\Gamma, \mathbf{k}_{\perp}=(0,0)\right]$ of plasmonic hole waveguides with square cross section of side length $s / a=0.54$.

results by the use of Fresnel's equations. ${ }^{56}$ If the energy is fixed and the incident angle varied, backbending will occur. On the other hand, if the incident angle is fixed and the energy varied, asymptotic behavior will appear. ${ }^{56}$ Further, if $k_{\|}$is considered complex and $\omega$ real, the dispersive curves bend back toward smaller $k_{\|}$as $\omega$ approaches $\omega_{\text {sp }}$, while if $\omega$ is considered complex and $k_{\|}$real, $k_{\|}$becomes infinite as $\omega$ approaches $\omega_{\mathrm{sp}} .{ }^{57}$

In the present study, the off-plane wave number $k_{\|}$is given as a parameter with real value and the eigenfrequency $\omega$ is obtained by solving a complex eigenvalue problem. The dispersion properties in the presence of loss in this study would therefore be referred to the latter case mentioned above. However, if, on the other hand, $\omega$ is given in real value and $k_{\|}$is solved in complex number, the dispersion curves of surface branches no longer asymptotically approach the surface plasma frequency $\omega_{\mathrm{sp}}$, as shown in Fig. 3 . In this situation, the crossing of the optical branch with the light line (cf. Fig. 4) might also be avoided, even in the case of $\varepsilon_{d}=1$ for the surrounding dielectric medium. Furthermore, the omnidirectional resonance and very slow group velocities (cf. Fig. 8) based on the asymptotic behavior at large $k_{\|}$do not hold in real systems.

\section{B. Interface condition and Rayleigh quotient}

The evanescent nature of surface plasmon modes can be identified by the discontinuity of the field slope across the interface, which is implied in the interface condition. For the in-plane case $\left(k_{\|}=0\right)$, the interface conditions (20) and (21) for TM and TE modes, respectively, are written as

$$
\begin{gathered}
{\left[\frac{\partial E_{\|}}{\partial n}\right]_{S}=0} \\
{\left[\frac{1}{\varepsilon} \frac{\partial H_{\|}}{\partial n}\right]_{S}=0 .}
\end{gathered}
$$

For TM modes, the $E_{\|}$field slope across the interface is continuous [cf. Eq. (42)], which indicates that no surface plas- 
mons exist for this polarization, while for TE modes, $1 / \varepsilon$ appears in the interface condition (43), accounting for the discontinuity of the $H_{\|}$field slope across the interface.

On the other hand, in the off-plane case $\left(k_{\|} \neq 0\right)$, the interface conditions become

$$
\begin{aligned}
& {\left[\frac{\varepsilon}{\Lambda \varepsilon-k_{\|}^{2}} \frac{\partial E_{\|}}{\partial n}\right]_{S}=0,} \\
& {\left[\frac{1}{\Lambda \varepsilon-k_{\|}^{2}} \frac{\partial H_{\|}}{\partial n}\right]_{S}=0,}
\end{aligned}
$$

for TM and TE modes, respectively. Appearance of $k_{\|}$in Eqs. (44) and (45) gradually changes the feature of surface plasmon modes. Note that $\partial E_{\|} / \partial n$ across the interface is no longer continuous and surface plasmons for TM modes are thus feasible. Besides, as $k_{\|}$increases, the interface conditions for TM and TE modes begin to reverse their natures. In Eq. (44), the role of $\varepsilon$ in the numerator becomes more dominant for larger $k_{\|}$, for the denominator tends to be equal on both sides of the interface. In the large $k_{\|}$limit, Eq. (44) acts like $\left[\varepsilon \partial E_{\|} / \partial n\right]_{S}=0$ and the discontinuity of the $E_{\|}$field slope is taken upon by $\varepsilon$, the signs of which being opposite on both sides of the interface. This is exactly how surface plasmon modes behave at the interface, thus also validating the existence of surface plasmon modes for TM polarization in the off-plane case. In fact, the basic features of surface plasmon modes for TM polarization in the off-plane case resemble those for TE polarization in the in-plane case, which have been addressed in Fig. 5. In the meanwhile, $\partial H_{\|} / \partial n$ in Eq. (45) tends to be continuous as $k_{\|}$increases to larger values. As a result, discontinuity of the $H_{\|}$field slope is smeared out for sufficiently large $k_{\|}$, and surface plasmon modes for TE polarization gradually grow into bulk modes.

It is also noticed that there are a large number of surface plasmon modes gathering around $\omega_{\mathrm{sp}}$ for TM polarization at sufficiently large $k_{\|}$, as those for TE polarization at $k_{\|}=0$. This high degenerate phenomenon can be explained with the help of the Rayleigh quotient. For a standard eigensystem $L \phi=\Lambda \phi$, the Rayleigh quotient is defined as

$$
R_{\phi}=\frac{\langle\phi, L \phi\rangle}{\langle\phi, \phi\rangle},
$$

where $\langle f, g\rangle=\int_{V_{\text {cell }}} f^{*} g d \tau$ is the inner product of $f$ and $g$ over the unit cell $V_{\text {cell }}$ with the asterisk symbol denoting the complex conjugate. It is known that the eigenvalue $\Lambda$ corresponds to the minimization of the Rayleigh quotient $R_{\phi}$ under the constraint that the corresponding eigenfunction $\phi$ be orthogonal to all previously obtained eigenfunctions. ${ }^{58}$

Based on the interface matching method in the preceding section, the Rayleigh quotient [Eq. (46)] can be rearranged using integration by parts in both the dielectric and the metal regions and applying the Bloch condition at the unit cell boundary to give

$$
R_{\phi}=\frac{S_{\phi}+V_{\phi}}{\varepsilon_{d} \int_{V_{d}}|\phi|^{2} d \tau+\int_{V_{m}}|\phi|^{2} d \tau},
$$

where

$$
\begin{gathered}
S_{\phi}=\int_{S} \phi^{*}\left[\frac{\partial \phi}{\partial n}\right]_{S} d a \\
V_{\phi}=\int_{V_{\text {cell }}}\left(|\nabla \phi|^{2}+k_{\|}^{2}|\phi|^{2}\right) d \tau+\Lambda_{p} \int_{V_{m}}|\phi|^{2} d \tau,
\end{gathered}
$$

in which $V_{d}$ and $V_{m}$ are the volumes of the dielectric and the metal, respectively, of the unit cell $V_{\text {cell }}$.

There are two important features implied in the Rayleigh quotient [Eq. (47)], pertaining to surface plasmon modes. The first feature is on the existence of surface plasmons. Appearance of $[\partial \phi / \partial n]_{S}$ in the surface integral term $S_{\phi}$ presents the contribution of the field slope discontinuity across the interface to the eigenfrequency and indicates the existence or subsistence of surface modes. Regarding the physical origin of surface plasmons, there should be surface charges to support electron oscillations in the metal. This connection is made clear through the transverse (in-plane) electric field components [recall Eqs. (7) and (9)]

$$
\begin{gathered}
\mathbf{E}_{\perp}^{\mathrm{TM}}=\frac{i k_{\|}}{(\omega / c)^{2} \varepsilon-k_{\|}^{2}} \nabla_{\perp} E_{\|}, \\
\mathbf{E}_{\perp}^{\mathrm{TE}}=-\frac{i \omega \mu_{0}}{(\omega / c)^{2} \varepsilon-k_{\|}^{2}} \hat{\mathbf{z}} \times \nabla_{\perp} H_{\|},
\end{gathered}
$$

for TM and TE modes, respectively. The surface charges are then evaluated by discontinuity of the in-plane electric field components across (and normal to) the interface,

$$
\begin{aligned}
\sigma^{\mathrm{TM}} & =\left[\mathbf{n} \cdot \mathbf{E}_{\perp}^{\mathrm{TM}}\right]_{S}, \\
\sigma^{\mathrm{TE}} & =\left[\mathbf{n} \cdot \mathbf{E}_{\perp}^{\mathrm{TE}}\right]_{S},
\end{aligned}
$$

where $\mathbf{n}$ is the outward unit vector normal to the interface $S$. Concerning either $k_{\|}=0$ or $\left[\partial E_{\|} / \partial n\right]_{S}=0$ for the in-plane case, it is evident that $\sigma^{\mathrm{TM}}=0$. On the other hand, $\sigma^{\mathrm{TE}} \neq 0$ even for $k_{\|}=0$, indicating that surface plasmons exist (and only exist) for TE modes in the in-plane case. On the other hand, for the off-plane problems $\left(k_{\|} \neq 0\right)$, both $\sigma^{\mathrm{TM}}$ and $\sigma^{\mathrm{TE}}$ are in general not zero. These charges are sources of surface plasma oscillations for periodic lattices of plasmonic waveguides. The difference between TM and TE polarizations is that $\sigma^{\mathrm{TE}}$ diminishes at large $k_{\|}$, while $\sigma^{\mathrm{TM}}$ remains to be nonzero, in view of the different expressions between Eqs. (48) and (49).

The second feature of the Rayleigh quotient [Eq. (47)] connecting to surface plasmon modes is on the degeneracy. Recall that the surface integral $S_{\phi}$ in the Rayleigh quotient $R_{\phi}$ contains only normal derivatives. It means that tangential variations of the field along the interface $S$ do not alter the value of $R_{\phi}$, and not the eigenfrequency either. Therefore, surface plasmon modes with the same frequency may consist of as many degenerate modes as possible, with mode structures that differ between one another only along the surface tangential direction. In principle, it is supposed to be infinite degeneracy for surface plasmon modes around the surface plasma frequency $\omega_{\text {sp. }}{ }^{20}$ This point is consistent with similar arguments for surface plasmon modes in plasmonic crystals, ${ }^{22}$ surface phonon modes in polaritonic crystals, ${ }^{30}$ as 
well as magnetic surface plasmon modes in negative index crystals. $^{31}$

\section{CONCLUDING REMARKS}

In conclusion, surface plasmon modes for periodic lattices of plasmonic hole waveguides were solved and analyzed. The interface matching method was proposed to solve the apparently nonlinear eigenvalue problem, with the proper use of the interface condition. Important characteristics of surface plasmon modes are implied in the interface condition, in which special features pertaining to off-plane surface plasmon modes were resolved. For periodic lattices of plasmonic waveguides, surface plasmon modes occur for both TE and TM polarizations, and as the off-plane wave number $k_{\|}$increases, their characteristics begin to interchange. On the one hand, a large number of static modes $(\omega=0)$ emerge at $k_{\|}=0$ for TM polarization. These modes gradually lose their bulk nature as $k_{\|}$increases and eventually they develop into surface plasmon modes with frequencies approaching the surface plasma frequency $\omega_{\text {sp }}$ in the large $k_{\|}$limit. On the other hand, the in-plane $\left(k_{\|}=0\right)$ surface plasmon modes for TE polarization gradually lose their surface nature and grow into bulk modes as $k_{\|}$increases. At large $k_{\|}$, the dispersion relations become parallel to the light line $\left(\omega=k_{\|} c\right)$ and the evanescent nature disappears. In addition, a particular TM optical branch appears for the hole waveguide structures, which intersects the light line and joins into the TM surface branches from above. With enough portion of plasmonic materials, the cutoff frequency of this optical branch is significantly higher than $\omega_{\mathrm{sp}}$ and the optical branch may exhibit substantial negative group velocities. In this situation, the net power flow through the unit cell along the plasmonic waveguides was shown to direct in the opposite direction of the wave vector.

\section{ACKNOWLEDGMENTS}

The author thanks Chien-Cheng Chang at National Taiwan University for helpful discussion. This work was supported in part by National Science Council of the Republic of China under Contracts No. NSC 96-2221-E-002-190MY3 and 96-2120-M-002-017.

\section{APPENDIX}

In this section, the inhomogeneous waveguide equations [Eqs. (3) and (4)] used in the present study are derived following a similar procedure for perfectly conducting waveguides. ${ }^{39}$ For time-harmonic electromagnetic fields (with $e^{-i \omega t}$ dependence), the Maxwell equations take the form

$$
\begin{gathered}
\nabla \times \mathbf{E}=i \omega \mu \mu_{0} \mathbf{H}, \\
\nabla \times \mathbf{H}=-i \omega \varepsilon \varepsilon_{0} \mathbf{E}, \\
\nabla \cdot\left(\mu \mu_{0} \mathbf{H}\right)=0,
\end{gathered}
$$

$$
\nabla \cdot\left(\varepsilon \varepsilon_{0} \mathbf{E}\right)=0
$$

for source-free problems. Let the waveguide geometry be constant in shape and size in the $z$ direction and assume that the fields vary as $e^{i k_{\|} z}$ along the $z$ axis. It is then useful to separate the fields into transverse and parallel components

$$
\begin{aligned}
& \mathbf{E}=\mathbf{E}_{\perp}+E_{\|} \hat{\mathbf{z}}, \\
& \mathbf{H}=\mathbf{H}_{\perp}+H_{\|} \hat{\mathbf{z}}
\end{aligned}
$$

and decompose the gradient as $\nabla=\nabla_{\perp}+\hat{\mathbf{z}} \partial / \partial z$. By taking $\hat{\mathbf{z}} \times$ on both sides of Eqs. (A1) and (A2), one can show that

$$
\begin{aligned}
i k_{\|} \mathbf{E}_{\perp}+i \omega \mu \mu_{0} \hat{\mathbf{z}} \times \mathbf{H}_{\perp} & =\nabla_{\perp} E_{\|}, \\
i k_{\|} \mathbf{H}_{\perp}-i \omega \varepsilon \varepsilon_{0} \hat{\mathbf{z}} \times \mathbf{E}_{\perp} & =\nabla_{\perp} H_{\|} .
\end{aligned}
$$

Performing $i k_{\|}$Eq. (A7) $-i \omega \mu \mu_{0} \hat{\mathbf{z}} \times$ Eq. (A8), one obtains

$$
\mathbf{E}_{\perp}=\frac{i}{\gamma^{2}}\left(k_{\|} \nabla_{\perp} E_{\|}-\omega \mu \mu_{0} \hat{\mathbf{z}} \times \nabla_{\perp} H_{\|}\right),
$$

where $\gamma^{2}=(\omega / c)^{2} \varepsilon \mu-k_{\|}^{2}$. Similarly, perform $i \omega \varepsilon \varepsilon_{0} \hat{\mathbf{z}} \times \mathrm{Eq}$. $(\mathrm{A} 7)+i k_{\|} \cdot$ Eq. $(\mathrm{A} 8)$ to give

$$
\mathbf{H}_{\perp}=\frac{i}{\gamma^{2}}\left(k_{\|} \nabla_{\perp} H_{\|}+\omega \varepsilon \varepsilon_{0} \hat{\mathbf{z}} \times \nabla_{\perp} E_{\|}\right) .
$$

For TM modes $\left(H_{\|}=0\right)$ and TE modes $\left(E_{\|}=0\right)$, respectively, Eqs. (A9) and (A10) are simplified to

$$
\begin{gathered}
\mathbf{E}_{\perp}=\frac{i k_{\|}}{\gamma^{2}} \nabla_{\perp} E_{\|}, \\
\mathbf{H}_{\perp}=\frac{i k_{\|}}{\gamma^{2}} \nabla_{\perp} H_{\|} .
\end{gathered}
$$

In the meanwhile, Eqs. (A3) and (A4) are written as

$$
\begin{gathered}
\nabla_{\perp} \cdot\left(\varepsilon \mathbf{E}_{\perp}\right)+i k_{\|} \varepsilon E_{\|}=0, \\
\nabla_{\perp} \cdot\left(\mu \mathbf{H}_{\perp}\right)+i k_{\|} \mu H_{\|}=0 .
\end{gathered}
$$

Inserting Eq. (A11) into Eq. (A13) and Eq. (A12) into Eq. (A14), one obtains the waveguide equations in the inhomogeneous medium,

$$
\begin{aligned}
& \nabla_{\perp} \cdot\left(\frac{\varepsilon}{\gamma^{2}} \nabla_{\perp} E_{\|}\right)+\varepsilon E_{\|}=0, \\
& \nabla_{\perp} \cdot\left(\frac{\mu}{\gamma^{2}} \nabla_{\perp} H_{\|}\right)+\mu H_{\|}=0,
\end{aligned}
$$

for TM and TE modes, respectively. For nonmagnetic materials $(\mu=1)$, Eq. (A16) is further simplified to

$$
\nabla_{\perp} \cdot\left(\frac{1}{\gamma^{2}} \nabla_{\perp} H_{\|}\right)+H_{\|}=0,
$$

with $\gamma^{2}=(\omega / c)^{2} \varepsilon-k_{\|}^{2}$. 
*chern@iam.ntu.edu.tw

${ }^{1}$ W. Barnes, A. Dereux, and T. Ebbesen, Nature (London) 424, 824 (2003)

${ }^{2}$ R. H. Ritchie, Phys. Rev. 106, 874 (1957).

${ }^{3}$ H. Ditlbacher, J. Krenn, B. Lamprecht, A. Leitner, and F. Aussenegg, Opt. Lett. 25, 563 (2000).

${ }^{4}$ G. Gbur, H. Schouten, and T. Visser, Appl. Phys. Lett. 87, 191109 (2005).

${ }^{5}$ K. Kneipp, Y. Wang, H. Kneipp, L. T. Perelman, I. Itzkan, R. R. Dasari, and M. S. Feld, Phys. Rev. Lett. 78, 1667 (1997).

${ }^{6}$ S. Nie and S. R. Emory, Science 275, 1102 (1997).

${ }^{7}$ W. H. Weber and C. F. Eagen, Opt. Lett. 4, 236 (1979).

${ }^{8}$ P. A. Hobson, S. Wedge, J. A. E. Wasey, I. Sage, and W. L. Barnes, Adv. Mater. (Weinheim, Ger.) 14, 1393 (2002).

${ }^{9}$ B. Hecht, H. Bielefeldt, L. Novotny, Y. Inouye, and D. W. Pohl, Phys. Rev. Lett. 77, 1889 (1996).

${ }^{10}$ J. Pendry, Science 285, 1687 (1999).

${ }^{11}$ P. Andrew, S. Kitson, and W. Barnes, J. Mod. Opt. 44, 395 (1997).

${ }^{12}$ M. Westphalen, U. Kreibig, J. Rostalski, H. Luth, and D. Meissner, Sol. Energy Mater. Sol. Cells 61, 105 (2000).

${ }^{13}$ S. C. Kitson, W. L. Barnes, and J. R. Sambles, Phys. Rev. Lett. 77, 2670 (1996).

${ }^{14}$ I. I. Smolyaninov, W. Atia, and C. C. Davis, Phys. Rev. B 59, 2454 (1999).

${ }^{15}$ H. Raether, Surface Plasmons on Smooth and Rough Surfaces and on Gratings (Springer, New York, 1988).

${ }^{16}$ F. Fujimoto and K. Komaki, J. Phys. Soc. Jpn. 25, 1679 (1968).

${ }^{17}$ R. Ruppin, Phys. Rev. B 11, 2871 (1975).

${ }^{18}$ C. A. Pfeiffer, E. N. Economou, and K. L. Ngai, Phys. Rev. B 10, 3038 (1974).

${ }^{19}$ S. S. Martinos and E. N. Economou, Phys. Rev. B 24, 6908 (1981).

${ }^{20}$ T. Ito and K. Sakoda, Phys. Rev. B 64, 045117 (2001).

${ }^{21}$ E. Moreno, D. Erni, and C. Hafner, Phys. Rev. B 65, 155120 (2002).

${ }^{22}$ R. L. Chern, C. C. Chang, and C. C. Chang, Phys. Rev. E 73, 036605 (2006).

${ }^{23}$ L. Novotny and C. Hafner, Phys. Rev. E 50, 4094 (1994).

${ }^{24}$ T. W. Ebbesen, H. J. Lezec, H. F. Ghaemi, T. Thio, and P. Wolff, Nature (London) 391, 667 (1998).

${ }^{25}$ J. A. Porto, F. J. Garcia-Vidal, and J. B. Pendry, Phys. Rev. Lett. 83, 2845 (1999).

${ }^{26}$ F. I. Baida and D. Van Labeke, Phys. Rev. B 67, 155314 (2003).

${ }^{27}$ H. Shin, P. B. Catrysse, and S. Fan, Phys. Rev. B 72, 085436 (2005).

${ }^{28}$ O. Toader and S. John, Phys. Rev. E 70, 046605 (2004).

${ }^{29}$ C. C. Chang, R. L. Chern, C. C. Chang, and R. R. Hwang, Phys. Rev. B 72, 205112 (2005).

${ }^{30}$ R. L. Chern, C. C. Chang, and C. C. Chang, Phys. Rev. B 73,
235123 (2006).

${ }^{31}$ R. L. Chern, C. C. Chang, and C. C. Chang, Phys. Rev. B 74, 155101 (2006).

${ }^{32} \mathrm{~W}$. Chew, Waves and Fields in Inhomogeneous Media (IEEE, New York, 1995).

${ }^{33}$ E. Economou, Phys. Rev. 182, 539 (1969).

${ }^{34}$ F. I. Baida, A. Belkhir, D. Van Labeke, and O. Lamrous, Phys. Rev. B 74, 205419 (2006).

${ }^{35}$ R. A. Ferrell, Phys. Rev. 111, 1214 (1958).

${ }^{36}$ G. Shvets, Phys. Rev. B 67, 035109 (2003).

${ }^{37}$ H. Shin and S. Fan, Phys. Rev. Lett. 96, 073907 (2006).

${ }^{38}$ P. Tournois and V. Laude, Opt. Commun. 137, 41 (1997).

${ }^{39}$ J. D. Jackson, Classical Electrodynamics, 3rd ed. (Wiley, New York, 1999).

${ }^{40}$ A. Yariv and P. Yeh, Optical Waves in Crystals (Wiley, New York, 1984).

${ }^{41}$ K. Sakoda, Optical Properties of Photonic Crystals (Springer, New York, 2004).

${ }^{42}$ C. G. B. Garrett and D. E. McCumber, Phys. Rev. A 1, 305 (1970).

${ }^{43}$ G. M. Gehring, A. Schweinsberg, C. Barsi, N. Kostinski, and R. W. Boyd, Science 312, 895 (2006).

${ }^{44}$ K. Aydin, K. Guven, C. M. Soukoulis, and E. Ozbay, Appl. Phys. Lett. 86, 124102 (2005).

${ }^{45}$ Y. Wang, Y. Zhang, L. He, F. Liu, H. Li, and H. Chen, J. Appl. Phys. 100, 113503 (2006).

${ }^{46}$ M. W. McCall, A. Lakhtakia, and W. S. Weiglhofer, Eur. J. Phys. 23, 353 (2002).

${ }^{47}$ R. Wangberg, J. Elser, E. E. Narimanov, and V. A. Podolskiy, J. Opt. Soc. Am. B 23, 498 (2006).

${ }^{48}$ F. Villa, T. Lopez-Rios, and L. E. Regalado, Phys. Rev. B 63, 165103 (2001).

${ }^{49}$ D. M. Pozar, Microwave Engineering, 3rd ed. (Wiley, New York, 2005).

${ }^{50}$ A. Karalis, E. Lidorikis, M. Ibanescu, J. D. Joannopoulos, and M. Soljačić, Phys. Rev. Lett. 95, 063901 (2005).

${ }^{51}$ H. Altug and J. Vučković, Appl. Phys. Lett. 86, 111102 (2005).

${ }^{52}$ M. Ibanescu, S. G. Johnson, D. Roundy, Y. Fink, and J. D. Joannopoulos, Opt. Lett. 30, 552 (2005).

${ }^{53}$ M. Soljacic and J. D. Joannopoulos, Nat. Mater. 3, 211 (2004).

${ }^{54}$ H. Shin, M. F. Yanik, S. Fan, R. Zia, and M. L. Brongersma, Appl. Phys. Lett. 84, 4421 (2004).

${ }^{55}$ E. T. Arakawa, M. W. Williams, R. N. Hamm, and R. H. Ritchie, Phys. Rev. Lett. 31, 1127 (1973).

${ }^{56}$ R. W. Alexander, G. S. Kovener, and R. Bell, Phys. Rev. Lett. 32, 154 (1974)

${ }^{57}$ G. S. Kovener, R. W. Alexander, Jr., and R. J. Bell, Phys. Rev. B 14, 1458 (1976).

${ }^{58}$ L. N. Trefethen and D. Bau, Numerical Linear Algebra (SIAM, Philadelphia, 1997). 